\title{
The Weak Mixing Angle from an $S U(3)$ Symmetry at a TeV
}

\author{
Savas Dimopoulos ${ }^{\mathrm{a}}$ and David Elazzar Kaplan ${ }^{\mathrm{b}}$

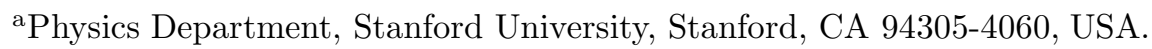 \\ ${ }^{\text {b}}$ SLAC, Stanford, CA 94025
}

\begin{abstract}
The measured values of two electroweak gauge couplings appear to obey an approximate (5\%) $S U(3)$ relation. Unless this is an accident caused by fortuitous Planck-scale physics, it suggests the presence of an $S U(3)$ symmetry near the electroweak scale. We propose this to be a local $S U(3)$ which spontaneously "mixes" with $S U(2) \times U(1)$ near a TeV. Although all the particles of the standard model are SU(3)-singlets, this symmetry relates the electroweak gauge couplings and can successfully predict the weak mixing angle with a precision of a few percent. Since this mechanism operates at a $\mathrm{TeV}$, it does not require an energy desert and consequently can be embedded in theories of TeV-gravity.
\end{abstract}

Introduction: The most impressive quantitative success of any extension of the standard model is the "unification" prediction of the weak mixing angle in the supersymmetric standard model (SSM) [1]: that is, a correlation between $\alpha_{s}\left(M_{Z}\right)$ and $\sin ^{2} \theta_{W}$, predicted in 1981 and confirmed by experiment at the two percent level [2] 10 years later. This has made the SSM the leading contender for physics beyond the standard model and supports the reality of a supersymmetric energy desert extending from a $\mathrm{TeV}$ to $10^{16}$ $\mathrm{GeV}$.

The reason for the enormity of the desert is the large disparity between the measured value of $\sin ^{2} \theta_{W}(=.231)$ [3] at the electroweak scale and its theoretical value at the fundamental (or unification) scale $(=3 / 8)$ [ 6 . This disparity necessitates a large hierarchy between the electroweak and unification scales, to allow $\sin ^{2} \theta_{W}$ to evolve from the experimental to its theoretical value.

In this paper we propose theories in which the theoretical value of $\sin ^{2} \theta_{W}$ is predicted to be near its experimental value at $M_{\mathrm{z}}$ and as a result have a fundamental scale near the electroweak energy - and no desert. Therefore in these theories a $\mathrm{TeV}$-scale cutoff, and therefore quantum gravity at a $\mathrm{TeV}$ [7], can coexist with a precise prediction of the weak mixing angle.
A key observation is that the electroweak gauge couplings and some of the matter content of the SM exhibit an approximate $S U(3)$ symmetry suggesting a more fundamental gauge sector at the weak scale. The Higgs doublet is contained in a triplet of $S U(3)$ and weak hypercharge is identified with the eighth generator of the $S U(3)$ [8,9]. The theory predicts $\sin ^{2} \theta_{W}=0.25$, close to the measured value of 0.231 , at the scale of $S U(3)$ breaking. The quarks, however, do not fit in this framework as hypercharges smaller than $1 / 2$ in magnitude are impossible to accommodate.

In this letter, we first present a simple extension to the SM which reproduces the approximate $S U(3)$ symmetry by correctly predicting $\sin ^{2} \theta_{W}$ to the few percent level. The well-known mechanism 10,11 which we coin "spontaneous mixing" is used to allow the SM sector to remain intact. We discuss the theoretical uncertainties in our model and compare to those of supersymmetric GUTs. Next we show how the mechanism can be easily embedded in theories at the weak scale which predict charge quantization, and we give two examples. Then we present an example of a model in which the Higgs is a pseudoGoldstone boson and the prediction of $\sin ^{2} \theta_{W}$ is maintained. We conclude with a brief discussion of experimental signatures and compare with other approaches. 
The Minimal Module: Consider the SM; add to it a new gauge group $S U(3)$ and a scalar $\Sigma$ which is a triplet of the new $S U(3)$, has the SM quantum number of the Higgs, $(2,-1 / 2)$ under $S U(2) \times U(1)$, and is a singlet of ordinary color. Let $\Sigma$ get a vacuum expectation value $(\mathrm{VEV})$ of the form:

$\langle\Sigma\rangle=\left(\begin{array}{cc}M & 0 \\ 0 & M \\ 0 & 0\end{array}\right)$,

breaking $S U(3) \times S U(2) \times U(1) \rightarrow S U(2) \times U(1)$. (For an explicit example which produces this VEV, see the Appendix.) At the scale $M$ the gauge couplings are related by:

$\frac{1}{g^{2}}=\frac{1}{g_{3}^{2}}+\frac{1}{\tilde{g}^{2}}$

and

$\frac{1}{g^{\prime 2}}=\frac{3}{g_{3}^{2}}+\frac{1}{\tilde{g}^{\prime 2}}$,

where $\left(g, g^{\prime}\right)$ are the gauge couplings of the standard electroweak theory, $\left(\tilde{g}, \tilde{g}^{\prime}\right)$ are the new $S U(2) \times U(1)$ couplings and $g_{3}$ is the $S U(3)$ gauge coupling. All couplings are evaluated at the scale $M$. Note, $g^{\prime}$ and $\tilde{g}^{\prime}$ are normalized such that the standard model particles have $U(1)$ charges equal to their canonical hypercharges (e.g., $Y=1 / 2$ for the lepton doublets). In the limit of large $\tilde{g}, \tilde{g}^{\prime}$, both low energy gauge couplings $g$ and $g^{\prime}$ are determined by the single $S U(3)$ coupling $g_{3}$. Therefore, although the SM sector has no $S U(3)$ symmetry, the low energy gauge couplings $g$ and $g^{\prime}$ at the scale $M$ are related by an (approximate) $S U(3)$ relation which leads to the value $\sin ^{2} \theta_{W} \simeq .25$. Using the renormalization group equations for the SM, one finds $\left.M_{o} \equiv M\right|_{\tilde{g}, \tilde{g}^{\prime} \rightarrow \infty}$ to be

$M_{o}=M_{\mathrm{z}} e^{-\frac{8 \pi^{2}}{b-b^{\prime} / 3}\left(g^{-2}-g^{\prime-2} / 3\right)}$.

Inserting the beta-function coefficients for the SM $\left(b, b^{\prime}\right)=(19 / 6,-41 / 6)$ and the value of the gauge couplings at $M_{\mathrm{z}}$, one finds $M_{o}=3.75 \mathrm{TeV}$. Therefore, in the exact $S U(3)$ limit, this is the prediction for the scale of $S U(3) \times S U(2) \times U(1)$ breaking to $S U(2) \times U(1)$.

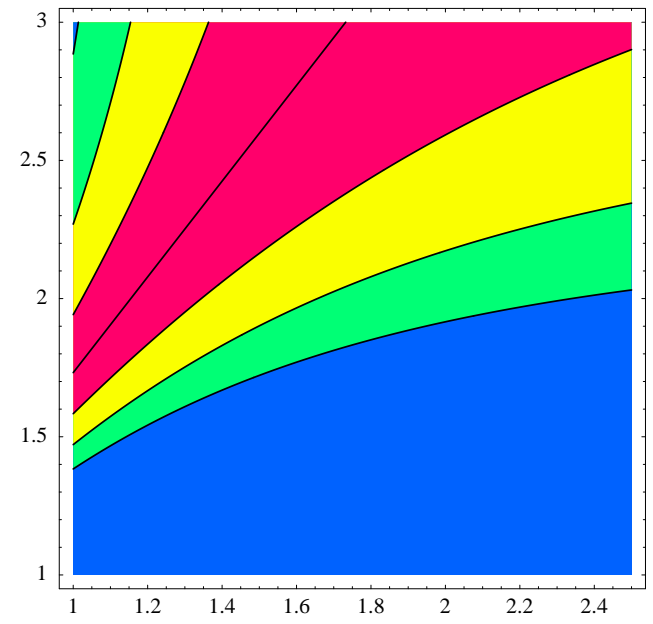

Figure 1. Contours of constant $\delta$ in the $\tilde{g}^{\prime}-\tilde{g}$ plane (see text). On the $\tilde{g}=\sqrt{3} \tilde{g}^{\prime}$ line there is no correction $(\delta=0)$. Going outward from this line are regions with $\delta<2 \%$ (red), $<4 \%$ (yellow), $<6 \%$ (green), and $>6 \%$ (blue).

Theoretical Uncertainty: In this section we quantify the main theoretical uncertainties in the module, namely the unknown values of the new gauge coupling $\tilde{g}$ and $\tilde{g}^{\prime}$ and the scale $M$. We then compare the status of this mechanism with that of normal supersymmetric grand unified theories.

The biggest source of uncertainty comes from the couplings $\tilde{g}$ and $\tilde{g}^{\prime}$. If these couplings are smaller than $g_{3}$, then the prediction for $\sin ^{2} \theta_{W}$ is completely washed out and $g$ and $g^{\prime}$ are dominated by the uncorrelated tilded gauge couplings. On the other hand, if $\tilde{g}, \tilde{g}^{\prime}$ are larger than $g_{3}$ then there is a significant region in parameter space in which the $S U(3)$ group dominates the prediction for $\sin ^{2} \theta_{W}$ and corrections to the $S U(3)$ symmetric relation are of order a few $\%$.

To measure the sensitivity of $\sin ^{2} \theta_{W}$ to $\tilde{g}$ and $\tilde{g}^{\prime}$, we fix $M$ to be the value computed in the previous section, $M_{o}=3.75 \mathrm{TeV}$. We then define

$\delta \equiv \frac{\delta \sin ^{2} \theta_{W}}{1 / 4}=\frac{3-\frac{g^{2}}{g^{\prime 2}}}{1+\frac{g^{2}}{g^{\prime 2}}}$ 


$$
=\frac{g(M)^{2}\left(\frac{3}{\tilde{g}^{\prime 2}}-\frac{1}{\tilde{g}^{2}}\right)}{4-g(M)^{2}\left(\frac{3}{\tilde{g}^{\prime 2}}-\frac{1}{\tilde{g}^{2}}\right)}
$$

where $g(M)$ is obtained by running the measured value of $g$ from $M_{\mathrm{z}}$ to $M$. The $\delta$ is the fractional deviation of $\sin ^{2} \theta_{W}$ from its $S U(3)$-symmetric value of .25 at $M$. In Figure 11, we plot values of $\delta$ as a function of $\tilde{g}$ and $\tilde{g}^{\prime}$. We limit the upper end of the parameter space to avoid the strongcoupling regime and Landau pole (we discuss the latter below). We see from the figure that in a significant portion (about $25 \%$ of the plotted region) of the parameter space with $\tilde{g}, \tilde{g}^{\prime}>1$ the deviation of $\sin ^{2} \theta_{W}$ from its $S U(3)$ value is less than $2 \%$.

Adding renormalization group running to eqs. (2) and (3) produces

$\frac{8 \pi^{2}}{g\left(M_{\mathrm{z}}\right)^{2}}=\frac{8 \pi^{2}}{g_{3}^{2}}-b \ln \frac{M_{o}}{M_{\mathrm{z}}}-b \ln \frac{M}{M_{o}}+\frac{8 \pi^{2}}{\tilde{g}^{2}}$,

and

$$
\frac{8 \pi^{2}}{g^{\prime}\left(M_{\mathrm{z}}\right)^{2}}=\frac{8 \pi^{2}}{g_{3}^{2} / 3}-b^{\prime} \ln \frac{M_{o}}{M_{\mathrm{z}}}-b^{\prime} \ln \frac{M}{M_{o}}+\frac{8 \pi^{2}}{\tilde{g}^{\prime 2}} .(7)
$$

We take the last two terms as the source of theoretical uncertainty of the module, where we have used $M_{o}$ as our "central value". Note that for $M$ in the range $1-10 \mathrm{TeV}$, its contribution to the fractional uncertainty is $\sim 3 \%$. Eliminating $g_{3}$, we can find the $S U(3)$-breaking scale $M$ as a function of $\left(\tilde{g}, \tilde{g}^{\prime}\right)$ :

$$
M=M_{\mathrm{z}} e^{\frac{8 \pi^{2}}{b-b^{\prime} / 3}\left[\left(\tilde{g}^{-2}-\tilde{g}^{\prime-2} / 3\right)-\left(g^{-2}-g^{\prime-2} / 3\right)\right]}
$$

where again the SM gauge couplings are evaluated at $M_{\mathrm{z}}$.

For obvious reasons, we do not extend the parameter space of the tilded gauge couplings to infinity. Even at semi-perturbative levels, there is an additional degree of uncertainty introduced due to the existence of a Landau pole in the $\tilde{g}^{\prime}$ coupling. This puts an upper limit on an ultraviolet cutoff $\Lambda$ in the theory. Unknown ultraviolet physics parameterized by operators such as

$$
\frac{|\Sigma|^{2}}{\Lambda^{2}} F^{\mu \nu} F_{\mu \nu},
$$

where $F^{\mu \nu}$ is the weak or hypercharge gauge bosons, can change the predicted value of $\sin ^{2} \theta_{W}$ at $M$. These effects limit the parameter space in the region of large $\tilde{g}^{\prime}$.

The location of the Landau pole is defined as the scale at which the coupling $\tilde{g}^{\prime}$ blows up from the effects of one-loop running:

$\Lambda=M e^{-\frac{8 \pi^{2}}{b_{\Sigma}^{\prime} \tilde{g}^{\prime 2}}}$

where the beta-function coefficient $b_{\Sigma}^{\prime}=-22 / 3$ includes the $\Sigma$ field in the $\left(3,2_{-1 / 2}\right)$ representation. For $\tilde{g}^{\prime}(M)=2.2$, the Landau pole is an order of magnitude above $M$ and operators like (9) contribute less than $1 \%$ uncertainty. For $\tilde{g}^{\prime}(M)=3.0$ the Landau pole is only a factor of 3.3 above $M$ and physics at the scale $\Lambda$ may affect the prediction of $\sin ^{2} \theta_{W}$.

Embedding the $U(1)$ into an asymptotically free group at $\sim M$ avoids the issue of the Landau pole. We discuss two such possibilities in the next section.

There are additional uncertainties coming from the normal threshold effects which will contribute to $\sin ^{2} \theta_{W}$ as well. In the minimal model, the unknown effects come from the Higgs and $\Sigma$ masses. For weak-scale Higgs masses, the Higgs threshold correction to $\sin ^{2} \theta_{W}$ is estimated to be less than two tenths of a percent [?]. The $\Sigma$ contribution can be estimated by allowing the mass of the remaining physical triplet (see the Appendix) to vary over a range of scales. It too has a relatively small effect for masses between 1 and $10 \mathrm{TeV}$.

Now let us compare to the SSM. Although the SSM has 125 parameters [12], its gauge sector (at one loop) is not contaminated by the vast flavor sector. This leads to the prediction of the weak mixing angle as follows. The experimental values of the three gauge couplings at $M_{\mathrm{z}}$ are determined by three theoretical parameters: the unification scale $M_{G U T}$, the value of the common gauge coupling $g_{G U T}$ at that scale, and the scale of superpartner masses $M_{s}$. Since the number of experimental and theoretical parameters are the same, we expect no real prediction. However, for small $M_{s}$, not too far from $M_{\mathrm{z}}$, the prediction of $\sin ^{2} \theta_{W}$ is sensitive to the value of $M_{s}$ only at the percent level. So, for a range of relatively small 
$M_{s}$, we obtain the successful $\sin ^{2} \theta_{W}$ prediction. It is, perhaps, more correct to view this as a prediction of the presence of light sparticles near $M_{\mathrm{z}}$, rather than a prediction of $\sin ^{2} \theta_{W}$. A reason why this is not viewed as "fitting $\sin ^{2} \theta_{W}$ by adding $\sim 100$ new particles" is that the presence of sparticles near $M_{\mathrm{z}}$ is independently motivated by the hierarchy problem.

In our present proposal the accounting is similar. For $\tilde{g}, \tilde{g} \prime$ in the broad range shown in the figure, the value of $\sin ^{2} \theta_{W}$ is independent of these at the few \% level and is determined by the $S U(3)$ symmetric dynamics encoded in $g_{3}$. Thus the experimental values of the two electroweak gauge couplings are determined in terms of the two parameters $g_{3}$ and $M$. For small $M$ not too far from $M_{\mathrm{z}}$, the prediction of $\sin ^{2} \theta_{W}$ depends logrithmically on, and is therefore insensitive to $M$. Just as in the SSM, we can view the smallness of the theoretical uncertainty, perhaps more correctly, as a prediction of concrete new physics at the scale $\mathrm{M} \sim$ few $\mathrm{TeV}$.

A fundamental distinction is that the SSM is an explicit model, whereas here we are proposing a mechanism (or module) which may be embedded in different models. The simplest models implementing our mechanism have a fine tuning of about one part in $10^{3}$ or $10^{4}$ to ensure that the Higgs remains lighter than the fundamental scale. In the SSM the fine tuning inferred from the absence of light sparticles is about $1 \%$. Combining supersymmetry with our mechanism would reduce the fine tuning to the same $1 \%$ level. We discuss another possibility in a later section.

Charge Quantization: One prediction common to all semi-simple grand unified theories is quantized hypercharge. The model described above contains a $U(1)$ factor and therefore does not require charges to be rational. The prediction of $\sin ^{2} \theta_{W}$ at $M$ is in fact dependent on a continuous parameter, the $U(1)$ charge of $\Sigma$. For a charge $-x$ in the same canonical normalization of SM hypercharges, the unbroken $U(1)$ gauge boson below the scale $M$ is

$B_{\mu}=\frac{\tilde{g}^{\prime} x A_{\mu}^{8}+\left(g_{3} / 2 \sqrt{3}\right) \tilde{B}_{\mu}}{\sqrt{g_{3}^{2} / 12+\tilde{g}^{\prime 2} x^{2}}}$,

where $A_{\mu}^{8}$ and $\tilde{B}_{\mu}$ are the $S U(3)$ "hypercharge" and $U(1)$ gauge bosons respectively. A SM field $\psi$ with $U(1)$ charge $\tilde{Y}$ will couple to $B_{\mu}$ as

$$
\begin{aligned}
i \tilde{Y} \tilde{g}^{\prime} \tilde{B}_{\mu} \psi & =\frac{i \tilde{Y} \tilde{g}^{\prime} g_{3} / 2 \sqrt{3}}{\sqrt{g_{3}^{2} / 12+\tilde{g}^{\prime 2} x^{2}}} B_{\mu}+\cdots \\
& \rightarrow i \tilde{Y} \frac{g_{3}}{2 \sqrt{3} x} B_{\mu}+\cdots \\
& \equiv i Y g^{\prime} B_{\mu}+\cdots
\end{aligned}
$$

where in the second line the limit $\tilde{g}^{\prime} \rightarrow \infty$ is taken. Having chosen charges such that $Y=\tilde{Y}$, we see that $g^{\prime}=g_{3} /(2 \sqrt{3} x)$ and

$\sin ^{2} \theta_{W}=\frac{1}{1+12 x^{2}}$ at $M$.

For continuous $x$ there exists a continuous set of predictions for $\sin ^{2} \theta_{W}$. However, if the scenario is embedded in a more fundamental theory where charge quantization is generic (i.e., string theory), the prediction of $\sin ^{2} \theta_{W}$ takes on more significance. The model may also be embedded in a field theory which predicts rational charges, such as a semi-simple Yang-Mills theory. Since the larger group manifests itself at low energies, it must not mediate proton decay. Two well known examples of such a group are Pati-Salam $\left(S U(4)_{c} \times S U(2)_{L} \times S U(2)_{R}\right)[5$ and trinification $\left(S U(3)_{c} \times S U(3)_{L} \times S U(3)_{R} / \mathcal{Z}_{3}\right)[13]$.

In Pati-Salam, there are two independent gauge couplings when the two $S U(2)$ coupling are set equal by imposing parity. The field content of the SM fits beautifully: the matter multiplets are the $(4,2,1)$ containing the quark and lepton doublets, the $(\overline{4}, 1,2)$ containing the quark and lepton singlets (including a right-handed neutrino), and a scalar $(1,2,2)$ containing two Higgs doublets. Under the Pati-Salam group, $\Sigma$ must transform as a multiplet which contains a doublet of charge $1 / 2$, e.g., $(1,2,2)$ or $(4,2,1)$.

The $S U(2) \times U(1)$ can also be embedded into $S U(3)^{3}$ (without the modded out $\mathcal{Z}_{3}$ factor). The gauge couplings of the $S U(3)_{L, R}$ portion can be made relatively strong (and equal by again imposing a parity symmetry) and the standard multiplets contain the SM fermions as the only chiral matter. The $\Sigma$ can be a $(3,1,3, \overline{3})$ under "quadrification": $S U(3)$-new, color, left and right respectively. 
In both Pati-Salam and quadrification additional breaking of gauge symmetries must occur. This can be done in the usual way for these groups [5.13] or may be incorporated with the breaking of the weak $S U(3)$. Some additional structure is required to avoid unwanted Yukawa relations and (too-)large neutrino masses. For example, in quadrification, fermion masses are unrelated if there are two Higgs multiplets - one for quarks and one for leptons. A complete description of models of this type will appear in future work 14.

Embedding the $U(1)$ group of the minimal module into semi-simple ones tend to widen the parameter space to which $\sin ^{2} \theta_{W}$ is insensitive. This is because after breaking the semi-simple group down to the strong $S U(2) \times U(1)$, the range of values for $\tilde{g}^{\prime} / \tilde{g}$ is smaller than the normal range (zero to infinity) due to the correlation of $\tilde{g}^{\prime}$ to other gauge couplings. In Pati-Salam, for instance, the effective $U(1)$ coupling $\tilde{g}^{\prime}$ is a function of $g_{4}$ and $g_{R}$ :

$\tilde{g}^{\prime}=\frac{g_{R} g_{4} \sqrt{3} /(2 \sqrt{2})}{\sqrt{g_{R}^{2} / 4+3 g_{4}^{2} / 8}}$

where $g_{4}$ at the breaking scale $M_{P S}$ is equal to the QCD coupling $g_{c}$ at $M_{P S}$, which can be determined by running its measured value up from $M_{\mathrm{z}}$. Note that for $g_{R} \rightarrow \infty, \tilde{g}^{\prime}$ does not blow up but hits the asymptotic value of $\sqrt{3 / 2} g_{4}$. In quadrification, $\tilde{g}^{\prime}$ is a function of the left and right $S U(3)$ couplings:

$\tilde{g}^{\prime}=\frac{g_{L} g_{R} / 2}{\sqrt{g_{R}^{2} / 12+g_{L}^{2} / 3}}$

Here in the limit $g_{R} \rightarrow \infty, \tilde{g}^{\prime} \rightarrow \sqrt{3} g_{L}$. In neither case does the ratio $\tilde{g}^{\prime} / \tilde{g}$ blow up anywhere in the parameter space.

Figures 2 and 3 are the analogs of Figure 1 for Pati-Salam and quadrification respectively. They are plotted in the plane of the $S U(2)_{L} \times S U(2)_{R}$ and $S U(3)_{L} \times S U(3)_{R}$ gauge couplings where we have dropped the parity restriction. We are not working from explicit models and for simplicity we assume that the larger group breaks to the minimum module at the same scale $M=3.75$ $\mathrm{TeV}$ as the weak $S U(3)$.

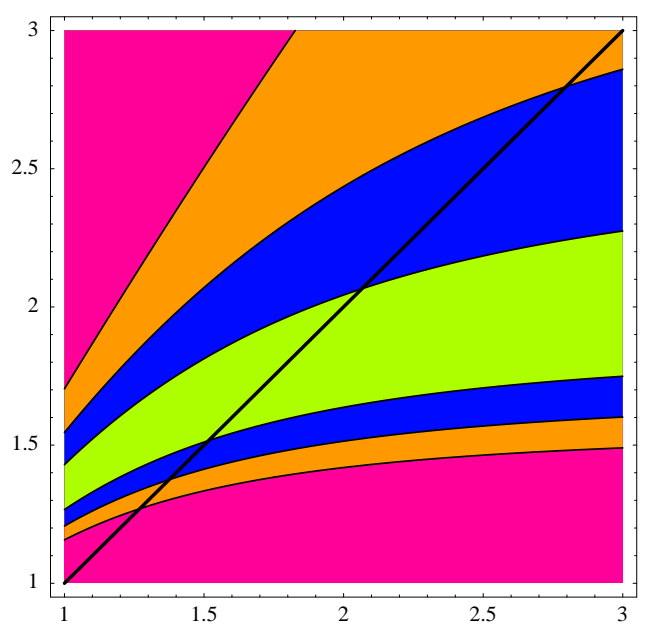

Figure 2. Contours of constant $\delta$ in the $g_{R^{-}} g_{L}$ plane for Pati-Salam. The thick black line corresponds to the parity-symmetric case. Going outward from the central region are regions with $\delta<2 \%$ (green), $<4 \%$ (blue), $<6 \%$ (orange), and $>6 \%$ (magenta).

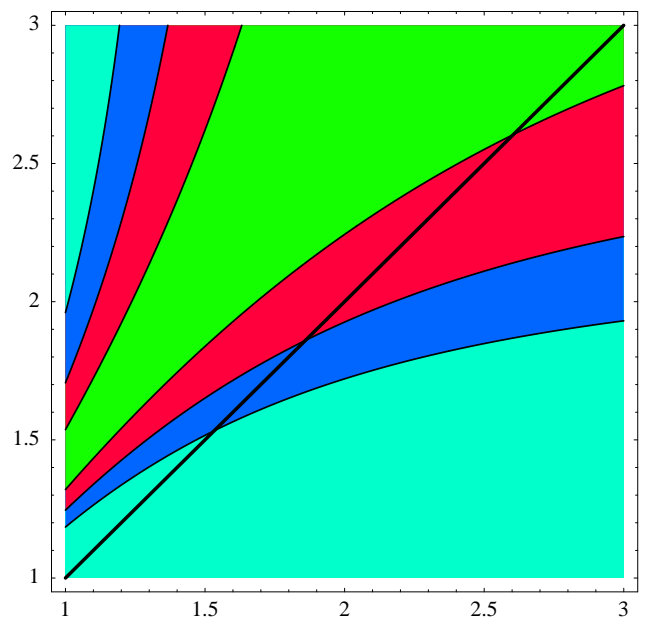

Figure 3. As in Figure 2, but for quadrification. Again the parity-symmetric case is shown. The colors outward from the central region are $\delta<2 \%$ (green), $<4 \%$ (red), $<6 \%$ (blue), and $>6 \%$ (aqua). 
We see from the figures that the effects on $\sin ^{2} \theta_{W}$ are small in a larger segment of the parameter space. Restoring parity as a symmetry restricts the parameter space to the diagonal line on each plot. On the parity-symmetric line in Figure $2 \sin ^{2} \theta_{W}$ is particularly insensitive - much of the line lies in the $<4 \%$ correction region.

The inclusion of the module into a semi-simple group produces three benefits: 1 . charge quantization, 2. removal of the Landau pole, and 3. enhancement of the insensitive region of parameter space. The examples we have presented are the well known groups into which SM matter fits perfectly, i.e., is the only chiral matter in the complete multiplets. Thus we are able to incorporate this success of standard GUTs. Constructing the complete models, taking into account all phenomenological consequences, would be a worthwhile endeavor.

The Hierarchy Problem: As mentioned before, the minimal module has a fine tuning of at least one part in $10^{3}$ associated with the Higgs mass. Of course, the module can easily be supersymmetrized to reduce the fine tuning. The purpose of this section is to present an example in which the hierarchy problem is ameliorated without the use of supersymmetry while maintaining the successful prediction of $\sin ^{2} \theta_{W}$. For our example, we choose a model in which the Higgs is a pseudo-Goldstone boson 15, 16]. We present a simple version of these models which leaves a fine-tuning of about $\sim 1 \%$. Versions with more structure along the lines of [16] can eliminate the fine-tuning completely.

The model has two sets of Nambu-Goldstone bosons which can be written as a non-linear sigma model in the standard form [17]:

$U=e^{i \pi_{u} / 2 f}$

$V=e^{i \pi_{v} / 2 f}$,

where for simplicity we have set the decay constants $f$ equal. Each multiplet transforms under an independent $S U(4)_{L} \times S U(4)_{R}$ chiral symmetry:

$U \rightarrow L U R^{\dagger} \quad V \rightarrow \mathcal{R} V \mathcal{L}^{\dagger}$.

The "pions" transform linearly (in the adjoint representation) under the symmetric combination

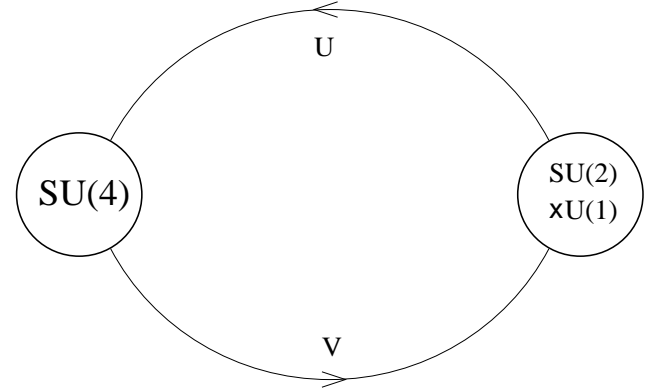

Figure 4. A diagrammatic description of the pseudo-Goldstone boson Higgs model. The two lines represent Goldstone bosons in the $(4, \overline{4})$ and $(\overline{4}, 4)$ of two sets of $S U(4)_{L} \times S U(4)_{R}$ chiral symmetries. The circles represent the gauging of the diagonal linear combination of the $S U(4)_{L}$ symmetries and an $S U(2) \times U(1)$ subgroup of the $S U(4)_{R}$ symmetries.

of their respective chiral symmetries and nonlinearly under the anti-symmetric combinations.

The chiral symmetries are broken by the following perturbations:

1. The diagonal combination of the two $S U(4)_{L}$ global symmetries is weakly gauged.

2. An $S U(2) \times U(1)$ subgroup of the diagonal combination of $S U(4)_{R}$ global symmetries is gauged somewhat more strongly than the $S U(4)_{L}$.

The model is represented by the diagram in Figure 4 .

Both $U$ and $V$ break the gauged $S U(4)$ and $S U(2) \times U(1)$ to a diagonal $S U(2) \times U(1)$ at the scale $f$. The $U(1)$ is chosen 1 such that the $S U(2) \times U(1)$ live in an $S U(3)$ subgroup of the diagonal $S U(4)_{R}$. Under the diagonal (fictitious) $S U(3)$ the pions transform as an $8+3+\overline{3}+1$. Choosing Higgs doublets with hypercharge $\pm 1 / 2$

\footnotetext{
${ }^{1}$ This may be a natural consequence of embedding the $S U(2) \times U(1)$ in a semi-simple group, e.g., Pati-Salam, broken at the cutoff and imposing some discrete symmetries.
} 
to live in the $3+\overline{3}$ gives the prediction $\sin ^{2} \theta_{W} \sim$ .25 at the scale $f$.

Now it is clear why we had to choose $S U(4)$ instead of $S U(3)$. The pseudo-Goldstone bosons come in adjoint representation of the vector part of the chiral symmetry. We require a group which contains an $S U(3)$ subgroup and whose adjoint representation contains a triplet. The smallest possibility is $G_{2}$ whose adjoint is a 14 and contains $8+3+\overline{3}$. Because it is rank 2 there are discrete choices for $S U(2) \times U(1)$. However, for the simplicity of this description, we have chosen the example more indicative of QCD.

The $S U(4)$-adjoint pions break down under $S U(2) \times U(1)$ to an uncharged triplet, two uncharged singlets, a complex charge-one singlet, a complex charge- $3 / 2$ doublet and a complex charge- $1 / 2$ doublet. The last of these can play the role of the SM Higgs.

The fate of the two sets of pions is as follows: one linear combination is eaten by the breaking of the gauge symmetry to the diagonal group while the other can be parameterized in unitary gauge as

$U * V \equiv \mathcal{U}=e^{i \pi / 2 f}$.

Note the scale $f$ is where $\sin ^{2} \theta_{W}$ exhibits the approximate $S U(3)$ relation. Therefore we take $f \sim$ few $\mathrm{TeV}$. The gauge couplings explicitly break the global symmetries of the theory and distinguish between different components of the pions. Therefore, we expect to generate operators at one loop which treat the remaining Goldstones differently. Some examples of operators are:

$$
\begin{array}{ll}
\operatorname{tr}\left[\mathcal{U} T^{a} \mathcal{U}^{\dagger} T^{a}\right] & \left|\operatorname{tr}\left[\mathcal{U} T^{a}\right]\right|^{2} \\
\operatorname{tr}\left[\mathcal{U} T^{8} \mathcal{U}^{\dagger} T^{8}\right] & \left|\operatorname{tr}\left[\mathcal{U} T^{8}\right]\right|^{2}
\end{array}
$$

where the $T \mathrm{~s}$ are $S U(4)$ generators and $a=$ $1,2,3$. These operators will give both quadratic and quartic couplings to the pions, with the different $S U(2) \times U(1)$ representations getting different masses. In addition, there will be couplings to fermions, most importantly the top quark, which distinguish between the pseudo-Goldstone bosons. It is hopeful that in some region of parameter space, the charge- $1 / 2$ doublet gets the (dominant) VEV and properly breaks electroweak symmetry. The natural scale for the VEV is $\sim f$ and so fine-tuning of about $1 \%$ is required to get the correct electroweak symmetry breaking scale relative to the higher $S U(3)$-breaking scale.

Additional structure like a two-by-two lattice of the type introduced in [16] can remove the fine tuning entirely 14. In such a model, the size of the VEV is naturally a loop smaller than the decay constant, or in other words, $v \sim f / 4 \pi$. Remarkably, this is just what is needed to run from the $S U(3)$ value of $\sin ^{2} \theta_{W}$ to the measured value. We postpone discussion of models of this type to a longer article [14].

Discussion: In this framework, the standard model is valid up to energies of order a few $\mathrm{TeV}$. At that scale new particles and phenomena begin to emerge; some are model independent and an integral part of the mechanism we are proposing. These include the extra gauge bosons associated with the full $S U(3) \times S U(2) \times U(1)$ as well as the $\Sigma$ multiplet that bridges the $S U(2) \times U(1)$ and $S U(3)$. Also, at a few $\mathrm{TeV}$, the $S U(2) \times U(1)$ forces become strong. What happens beyond that is model dependent. Some new physics must set in to protect the theory from the $U(1)$ Landau pole and to account for charge quantization. Our favorite possibility is that full string theory emerges at a few TeV. Other, more explicit possibilities - such as Pati-Salam, quadrification or composite Higgs models- have already been discussed in previous sections. In each case there is rich new physics to look forward to.

There are at least three other approaches to computing $\sin ^{2} \theta_{W}$ in theories with string scale near a $\mathrm{TeV}$. The earliest [18 relies on accelerating the normal evolution of gauge couplings [1] by having $\mathrm{TeV}^{-1}$ size extra dimensions. A potential obstacle to this approach is that the values of higher dimensional gauge couplings are very sensitive to unknown short-distance physics. Another approach, valid for two large dimensions, exploits the mapping between ordinary RG-evolution and the profile of 2-D bulk fields It would be interesting to construct a realistic model based on this. Another proposal [21] introduces $\sim 13$ copies of the gauge and Higgs sector of the SSM. This reproduces the prediction of the SSM, though with significant theoretical 
uncertainty $\sim 20 \%$ - due to the threshold effects from each of the sectors.

These three proposals rely essentially on the same numerical reasons as the SSM to reproduce the value for $\sin ^{2} \theta_{W}$. In this paper we presented a different way to derive the value of $\sin ^{2} \theta_{W}$, unrelated to the SSM. It involves a low-energy $S U(3)$ symmetry whose presence can be directly tested at the LHC.

Acknowledgments: We are happy to thank Nima Arkani-Hamed, Lawrence Hall, David B. Kaplan, Steven Shenker and Yonathan Zunger for valuable discussions. This work is supported by NSF grant PHY-9870115 and DOE grant DEAC03-76SF00515.

\section{REFERENCES}

1. S. Dimopoulos and H. Georgi, Nucl. Phys. B 193, 150 (1981).

2. For a recent analysis, see D. M. Ghilencea and G. G. Ross, Nucl. Phys. B 606, 101 (2001) arXiv:hep-ph/0102306.

3. D. E. Groom et al. [Particle Data Group Collaboration], Eur. Phys. J. C 15, 1 (2000).

4. H. Georgi and S. Glashow, Phys. Rev. Lett. 327 (1974) 438;

H. Georgi, H. Quinn and S. Weinberg, Phys. Rev. Lett. 33 (1974) 451.

5. J. C. Pati and A. Salam, Phys. Rev. D 10, 275 (1974).

6. S. Dimopoulos, S. Raby and F. Wilczek, Phys. Rev. D 24, 1681 (1981).

7. N. Arkani-Hamed, S. Dimopoulos and G. R. Dvali, Phys. Lett. B 429, 263 (1998) arXiv:hep-ph/9803315;

I. Antoniadis, N. Arkani-Hamed, S. Dimopoulos and G. R. Dvali, Phys. Lett. B 436, 257 (1998) arXiv:hep-ph/9804398;

N. Arkani-Hamed, S. Dimopoulos and G. R. Dvali, Phys. Rev. D 59, 086004 (1999) arXiv:hep-ph/9807344.

8. S. Weinberg, Phys. Rev. D 5, 1962 (1972).

9. F. Pisano and V. Pleitez, Phys. Rev. D 46, 410 (1992) arXiv:hep-ph/9206242];/ P. H. Frampton, Phys. Rev. Lett. 69, 2889 (1992).

10. T. Yanagida, Phys. Lett. B 344, 211 (1995)
arXiv:hep-ph/9409329.

11. N. Weiner, arXiv:hep-ph/0106097.

12. S. Dimopoulos and D. W. Sutter, Nucl. Phys. B 452, 496 (1995) arXiv:hep-ph/9504415.

13. A. de Rújula, H. Georgi, and S.L. Glashow, in Fifth Workshop on Grand Unification, edited by K. Kang, H. Fried, and P. Frampton (World Scientific, Singapore, 1984), p. 88.

14. S. Dimopoulos and D. E. Kaplan, work in progress.

15. D. B. Kaplan and H. Georgi, Phys. Lett. B 136, 183 (1984).

16. N.Arkani-Hamed,

A. G. Cohen and H. Georgi, Phys. Lett. B 513, 232 (2001) arXiv:hep-ph/0105239.

17. For a review see Weak Interactions and Modern Particle Theory, H. Georgi, Benjamin/Cummings Publishing Co., Inc. (1984).

18. K. R. Dienes, E. Dudas and T. Gherghetta, Phys. Lett. B 436, 55 (1998) arXiv:hepph/9803466].

19. C. P. Bachas, JHEP 9811, 023 (1998) arXiv:hep-ph/9807415; / /

20. I. Antoniadis and C. Bachas, Phys. Lett. B 450, 83 (1999) arXiv:hep-th/9812093;// N. Arkani-Hamed, S. Dimopoulos and J. March-Russell, arXiv:hep-th/9908146.

21. N. Arkani-Hamed, A. G. Cohen and H. Georgi, arXiv:hep-th/0108089.

Appendix - Diagonal Breaking and the Potential: The most general renormalizable potential for $\Sigma$,

$\mathcal{V}=\lambda\left(\operatorname{tr} \Sigma^{\dagger} \Sigma-v^{2}\right)^{2}-\lambda^{\prime} \operatorname{det} \Sigma^{\dagger} \Sigma+$ const.

can produce the correct symmetry breaking $S U(3) \times S U(2) \times U(1) \rightarrow S U(2) \times U(1)$. To see this - and the resulting spectrum - we parameterize $\Sigma$ as follows:

$$
\begin{gathered}
\Sigma=e^{i \pi^{a} \lambda^{a} / 2 M} \times \\
\left(\begin{array}{cc}
\left(\eta_{0}+M\right)+\eta_{3} & \eta_{1}-i \eta_{2} \\
\eta_{1}+i \eta_{2} & \left(\eta_{0}+M\right)-\eta_{3} \\
0 & 0
\end{array}\right) e^{i \pi^{a^{\prime}} \sigma^{a^{\prime}} / 2 M}
\end{gathered}
$$

where $M$ is the scale of symmetry breaking and $\lambda$, $\sigma$ are Gell-Mann and Pauli matrices respectively, $a=1-8$ and $a^{\prime}=1,2,3,8$. We identify $\sigma^{8}$ with 
the identity matrix of size two. Of the twelve degrees of freedom in the complex $\Sigma$, eight are Nambu-Goldstone bosons (the $\pi \mathrm{s}$ ) eaten by the broken gauge generators. The potential for the remaining Higgs fields is

$$
\begin{gathered}
\mathcal{V}=\lambda\left(\left(\eta_{0}+M\right)^{2}+\eta^{2}-v^{2}\right)^{2} \\
-\quad \lambda^{\prime}\left(\left(\eta_{0}+M\right)^{2}-\eta^{2}\right)^{2},
\end{gathered}
$$

with $\eta=\left\{\eta_{1}, \eta_{2}, \eta_{3}\right\}$. If $\lambda>\lambda^{\prime}>0$, there is a discrete minimum for which $M=\sqrt{\lambda /\left(\lambda-\lambda^{\prime}\right)} v$ and a VEV of the form (1) is reproduced. The $S U(2)$ triplet $\eta$ gets a positive squared mass at tree level of size $m^{2}=4 \lambda \lambda^{\prime} v^{2} /\left(\lambda-\lambda^{\prime}\right)$. The singlet $\eta_{0}$ stays massless at tree level, but has a quartic coupling which induces a mass at one loop. 